\title{
Reversible magnetization below Tc in high-quality
}

\section{superconducting ceramics}

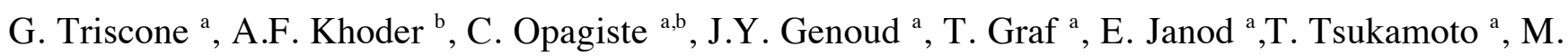
Couach $^{\text {b }}$, A. Junod ${ }^{\text {a }}$ J. Muller ${ }^{\text {a }}$

a Département de Physique de la Matière Condensée, Université de Genève, 24 quai Ernest-Ansermet, CH-1211 Genève 4, Switzerland

b CEA/Département de Recherche Fondamentale sur la Matière Condensée/SPSMS/LCP, 38054 Grenoble Cedex 9, France

\begin{abstract}
We have investigated the reversible magnetization below Tc in high-quality $\mathrm{YBa}_{2} \mathrm{Cu}_{3} \mathrm{O}_{7-d}(\mathrm{Y}-123), \mathrm{YBa}_{2} \mathrm{Cu}_{4} \mathrm{O}_{\mathrm{s}}$ (Y-124), $\mathrm{Y}_{2} \mathrm{Ba}_{4} \mathrm{Cu}_{7} \mathrm{O}_{15+\mathrm{x}}(\mathrm{Y}-247)$ and $\mathrm{Bi}_{2} \mathrm{Sr}_{2} \mathrm{CaCu}_{2} \mathrm{O}_{8+\mathrm{x}}$ (Bi-2212), $\mathrm{Tl}_{2} \mathrm{Ba}_{2} \mathrm{Cu}_{1} \mathrm{O}_{6+\mathrm{d}}(\mathrm{Tl}-2201)$ and $\mathrm{Tl}_{2} \mathrm{Ba}_{2} \mathrm{Ca}_{1} \mathrm{Cu}_{2} \mathrm{O}_{8+\mathrm{d}}$ (T1-2212) ceramics. Except for the stoichiometric Y-124 phase, the oxygen concentration was optimized in order to obtain the highest value of the critical temperature for which the normal-state susceptibility becomes temperature independent. Using the simple London model, we are able to fit the reversible magnetization $\mathrm{M}(\mathrm{T}$, $\mathrm{H}$ ) outside the region near Tc with good accuracy for the nearly three-dimensional $\mathrm{YBaCuO}$ phases. For the very anisotropic $\mathrm{BiSrCaCuO}$ and $\mathrm{TlBaCaCuO}$ phases, we have to include an additional term to take into account the fluctuations of vortices. An important result is that Y-123 exhibits a critical field clearly higher than those of the $\mathrm{BiSrCaCuO}$ or $\mathrm{TlBaCaCuO}$ phases. We obtain for the $\mathrm{Y}-123$ phase a slope $\mu_{0} \partial H_{\mathrm{c} 2, \mathrm{c}} /\left.\partial T\right|_{T_{\mathrm{c}}} \approx-4.3 \mathrm{~T} / \mathrm{K}$ and an extrapolated $\mu \mathrm{oH}_{\mathrm{C} 2, \mathrm{C}}(0)=280 \mathrm{~T}$.
\end{abstract}

\section{Introduction}

The measurement of the reversible magnetization of type-II superconductors is one of the methods to determine superconducting parameters such as the penetration depth $\lambda$, the coherence length $\xi$ and hence the critical fields and the Ginzburg-Landau parameter $\kappa$.

In superconductors with large $\kappa$, we can expect a large field domain $\left(\mathrm{H}_{\mathrm{C} 1}<\mathrm{H}<<\mathrm{H}_{\mathrm{C} 2}\right)$, where $\mathrm{M}$ depends logarithmically on the applied field (London regime). Recently, Hap and Clem [1,2] found that the London model is quantitatively incorrect. With their variational approach, where the core energy of vortices is taken into account, the magnetization turns out to still have an apparent $\ln H$ dependence but with two "new" somewhat field-dependent parameters.
For the HTSC compounds, the large anisotropy associated with the short coherence length and the high Tc introduces a more serious possibility of discrepancy between the London approach and the experimental $\mathrm{M}(\mathrm{T}, \mathrm{H})$ data. Thermal-fluctuation effects are enhanced in such quasi 2D structures above Tc as well as in the mixed state below To, where the entropy of vortex excitations (called "vortons" after Bulaevskii et al. [3]) becomes important and the equilibrium vortex density has to be renormalized by this dynamic contribution. The most spectacular result of these considerations is the existence, below To, of a crossing point $\left(T^{*}, M^{*}\right)$ where $M=M^{*}\left(T^{*}\right)$ is field independent [3-10].

In this paper, we use the above theories to fit the reversible magnetization below Tc for six selected high-Tc cuprates, i.e. three compounds characterized by a very large anisotropy, $\mathrm{Bi}_{2} \mathrm{Sr}_{2} \mathrm{CaCu}_{2} \mathrm{O}_{8+\mathrm{d}} \quad$ (Bi-2212), 
$\mathrm{T}_{2} \mathrm{Ba}_{2} \mathrm{CuO}_{6+\mathrm{X}}(\mathrm{T} 1-2201), \mathrm{TI}_{2} \mathrm{Ba}_{2} \mathrm{CaCu}_{2} \mathrm{O}_{8+\mathrm{z}}(\mathrm{T} 1-$ 2212), and three others exhibiting a rather more three-dimensional character, $\mathrm{YBa}_{2} \mathrm{Cu}_{3} \mathrm{O}_{7-\mathrm{X}}(\mathrm{Y}-$ $123), \mathrm{Y}_{2} \mathrm{Ba}_{4} \mathrm{Cu}_{7} \mathrm{O}_{15+\mathrm{d}}(\mathrm{Y}-247)$, and $\mathrm{YBa}_{2} \mathrm{Cu}_{4} \mathrm{O}_{8}$ $(\mathrm{Y}-124)$.

\section{Experimental details}

In the present analysis, we use the c.g.s, system where $\mathrm{B}[\mathrm{G}]=\mathrm{H}[\mathrm{Oe}]+4 \pi \mathrm{M}[\mathrm{G}]$ and $\chi v=M\left[\mathrm{emu} / \mathrm{cm}^{3}\right] / \mathrm{H}=\rho\left[\mathrm{g} / \mathrm{cm}^{3}\right] \chi \mathrm{g}[\mathrm{emu} / \mathrm{g}]$.

The Meissner effect (field cooling) was measured using a SQUID magnetometer with an external magnetic field of 20 Oe. The Meissner flux expulsion ratio $\mathrm{f}=-4 \pi \chi v$ was evaluated using an effective sample volume given by $\mathrm{m} / \mathrm{\rho}$ where $m$ is the mass and $\rho$ the X-ray density. A geometric demagnetization factor $\mathrm{D}$ was taken into account for all samples according to their approximate shape. The corrected susceptibility reads $\chi_{g}=\chi_{g}{ }^{m} /\left(1-4 \pi \mathrm{D} \chi_{g}{ }^{m}\right)$, where $\chi_{g}{ }^{m}$ is the measured susceptibility. The external magnetic field was calibrated using a high-purity superconducting $\mathrm{Pb}$ sphere.

The reversible magnetization $M(H, T)$ was measured with the same magnetometer using a small scan length $(3-4 \mathrm{~cm})$ to minimize the variation of the magnetic field during the displacement of the sample in the detection coils. With large magnetic field $(\geq 3-5 \mathrm{kOe})$ and below Tc, zero field cooling (ZFC) followed by field cooling (FC) measurements or else hysteresis $\mathrm{M}(\mathrm{H}) \mathrm{I}_{\mathrm{T}}$ measurements show especially for the anisotropic phases a large reversible temperature domain of about 30 to $40 \mathrm{~K}$ where thermodynamic relations are applicable. All measurements presented in the following were corrected by the normal-state contribution which has been obtained by a fit of the quasi field-independent normal-state susceptibility ) $\chi_{g}^{\text {normal }}(\mathrm{T})$ from $\mathrm{Tc}+30 \mathrm{~K}$ to room temperature. The magnetization is $\mathrm{M}(\mathrm{T})=\mathrm{M}^{\text {measured }}(\mathrm{T})-\chi_{g}^{\text {normal }}(\mathrm{T}) \rho H$.

\section{Sample preparation}

The preparation of the ceramics was described in previous papers. The listing of the phases, sample codes and the references is given in Table 1. Note that except for the stoichiometric Y-124 phase, the oxygen concentration was optimized in order to obtain the highest values of Tc where the normal-state susceptibility becomes temperature independent [11-14]. Table 2 gives a summary of the physical parameters $(T c, f$, space group, lattice constants and the average interlayer distance $s^{C}$ between superconducting or groups of superconducting planes).

\section{Model for the reversible magnetization below Tc}

Based on the Ginzburg-Landau (G-L) theory, the reversible magnetization $\mathrm{M}(\mathrm{H}, \mathrm{T})$ for superconducting materials with large $\kappa=\lambda / \xi$ $\left(\kappa \approx 10^{2}\right)$, a characteristic of cuprates, can be described by the London model. In this approach, where the order parameter $|\Psi|$ is assumed to be spatially constant and the vortex cores are treated as singularities, the reversible isothermal magnetization (no pinning) follows a logarithmic field dependence. This model is only valid for intermediate fields, i.e. $\left(\mathrm{H}_{\mathrm{C} 1}<<\mathrm{H}\right.$ $<<\mathrm{H}_{\mathrm{C} 2}$ ), (the so called London regime) [15], and predicts:

$$
\begin{aligned}
& -4 \pi M(T, H) \\
& =\frac{\Phi_{0}}{8 \pi \lambda^{2}(T)} \ln \left(\frac{\eta}{e} \frac{H_{\mathrm{c} 2}(T)}{H}\right), \quad \text { (c.g.s. units) }
\end{aligned}
$$

where $\eta$ is a constant depending on the vortex structure and $\phi_{\mathrm{o}}$ the flux quantum $(\hbar \mathrm{c} / 2 \mathrm{e}=$ $2.0679 \times 10^{-7} \mathrm{G} . \mathrm{cm}^{2}$ ).

In the following subsections, we evaluate the magnetization for anisotropic ceramics using the effective- mass model.

\section{1. Superconducting phases with large anisotropy}

In the case of ceramics with large anisotropy $\gamma^{2}=M_{c} / M_{a b}=\lambda_{c}{ }^{2} / \lambda_{a b}{ }^{2} \gg 25$ where Mi and $\lambda \mathrm{i}$ are the effective mass and the penetration depth along the i direction, respectively; we consider only the projection of the magnetization in the $\mathrm{c}$ direction, $\mathrm{m}_{\mathrm{c}}$, along the applied field $\mathrm{H}$. The measured magnetization $<M(T, H)>$ is obtained after integration over all grain orientations:

$$
\begin{aligned}
& -4 \pi\langle M(T, H)\rangle \\
& \quad=\frac{1}{2} \frac{\Phi_{0}}{8 \pi \lambda_{a b}^{2}(T)} \ln \left(\sqrt{\mathrm{e}} \frac{\eta}{\mathrm{e}} \frac{\mathrm{H}_{\mathrm{c} 2, c}(\mathrm{~T})}{\mathrm{H}}\right) .
\end{aligned}
$$


Table 1:

\begin{tabular}{llll}
\hline Phase & Stoichiometry & Code & Ref. \\
\hline Y-123a & $\mathrm{Y}: \mathrm{Ba}: \mathrm{Cu}=1: 2: 3$ & CR2b460 & {$[5 \mathrm{l}]$} \\
$\mathrm{Y}-123 \mathrm{~b}$ & $\mathrm{Y}: \mathrm{Ba}: \mathrm{Cu}=1: 2: 3$ & J465b & {$[52,53]$} \\
$\mathrm{Y}-124$ & $\mathrm{Y}: \mathrm{Ba}: \mathrm{Cu}=1: 2: 4$ & YlC52C & {$[54]$} \\
$\mathrm{Y}-247 \mathrm{a}$ & $\mathrm{Y}: \mathrm{Ba}: \mathrm{Cu}=2: 4: 7$ & G2al6 & {$[55]$} \\
$\mathrm{Y}-247 \mathrm{~b}$ & $\mathrm{Y}: \mathrm{Ba}: \mathrm{Cu}=2: 4: 7$ & $247-178$ & {$[55]$} \\
Bi-2212a & $\mathrm{Bi}: \mathrm{Sr}: \mathrm{Ca}: \mathrm{Cu}=2: 2: 1: 2$ & $\mathrm{~A}-3-600$ & {$[12]$} \\
$\mathrm{Bi}-2212 \mathrm{~b}$ & $\mathrm{Bi}: \mathrm{Sr}: \mathrm{Ca}: \mathrm{Cu}=2.12: 1.9: 1.02: 1.96$ & $\mathrm{~N} /-2 / 6$ & {$[56]$} \\
$\mathrm{T} 1-2201 \mathrm{a}$ & $\mathrm{Tl}: \mathrm{Ba}: \mathrm{Ca}: \mathrm{Cu}=2: 2: 0: 1$ & $12 \mathrm{~A} 0$ & {$[57]$} \\
$\mathrm{Tl}-2201 \mathrm{~b}$ & $\mathrm{Tl}: \mathrm{Ba}: \mathrm{Ca}: \mathrm{Cu}=2: 2: 0: 1$ & TA2-850 & {$[58]$} \\
$\mathrm{Tl}-2212$ & $\mathrm{Tl}: \mathrm{Ba}: \mathrm{Ca}: \mathrm{Cu}=1.8: 2: 1: 2$ & T2-13 & {$[59]$} \\
\hline
\end{tabular}

Table 2:

\begin{tabular}{|c|c|c|c|c|c|c|c|}
\hline Phase & $T_{\mathrm{c}}^{\text {onset }}(\mathrm{K})$ & $f(\%)$ & Space group & $a(\AA)$ & $b(\AA)$ & $c(\AA)$ & $s^{\mathrm{c}}(\AA)$ \\
\hline$Y-123 a$ & 92.3 & 41 & P mmm & $3.8170(5)$ & $3.8855(4)$ & $11.685(1)$ & 11.7 \\
\hline$Y-123 b$ & 91.7 & 68 & P mmm & $3.8173(5)$ & $3.8861(6)$ & $11.679(1)$ & 11.7 \\
\hline$Y-124$ & 80.1 & 39 & A mmm & $3.8428(5)$ & $3.8707(4)$ & $27.242(3)$ & 13.6 \\
\hline$Y-247 a^{a}$ & 94.2 & 45 & A mmm & $3.8322(7)$ & $3.8770(6)$ & $50.600(6)$ & 12.7 \\
\hline$Y-247 b$ & 94.8 & 36 & A mmm & $3.8311(6)$ & $3.8773(5)$ & $50.591(8)$ & 12.7 \\
\hline $\mathrm{Bi}-2212 \mathrm{a}$ & 93.7 & 32 & A2aa & $5.409(1)$ & $5.411(1)$ & $30.90(1)$ & 15.5 \\
\hline $\mathrm{Bi}-2212 \mathrm{~b}$ & 92.4 & 48 & A2aa & $5.410(1)$ & $5.412(2)$ & $30.88(1)$ & 15.5 \\
\hline Tl-2201a & 92.1 & 61 & $\mathrm{I} 4 / \mathrm{mmm}$ & \multicolumn{2}{|c|}{$a=b=3.8700(3)$} & $23.226(2)$ & 11.6 \\
\hline Tl-2201b & 92.1 & 63 & $\mathrm{I} 4 / \mathrm{mmm}$ & \multicolumn{2}{|c|}{$a=b=3.8706(4)$} & $23.224(3)$ & 11.6 \\
\hline $\mathrm{Tl}-2212$ & 108.7 & 45 & $\mathrm{I} 4 / \mathrm{mmm}$ & \multicolumn{2}{|c|}{$a=b=3.8548(5)$} & $29.328(4)$ & 14.7 \\
\hline
\end{tabular}

$T_{\mathrm{c}}^{\text {onset }}=0.1 \%$ of perfect Meissner state.

\subsection{Superconducting phases with intermediate anisotropy}

On the basis of the work of Kogan et al. [16], the measured magnetization for a ceramic is calculated in the appendix, with the result :

$$
\begin{aligned}
-4 \pi & \langle M(T, H)\rangle \\
& =\frac{\Phi_{0}}{8 \pi \lambda_{a b}^{2}(T)} \frac{I(\gamma)}{\gamma} \ln \left(e(\gamma) \frac{\eta}{e} \frac{H_{\mathrm{c} 2, c}(T)}{H}\right) .
\end{aligned}
$$
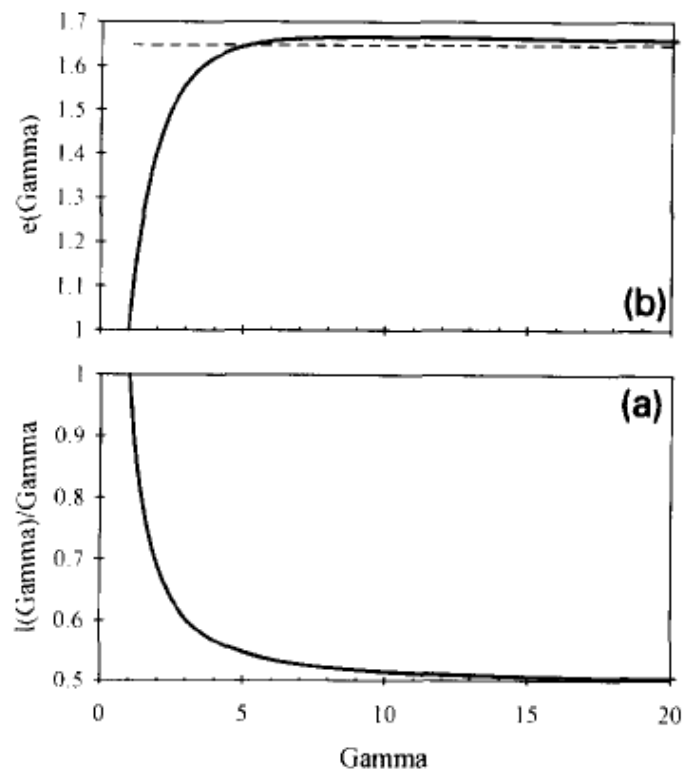

Fig.1 $: I(\gamma) / \gamma($ a) and $e(\gamma)(b)$ as a function of the anisotropy $\gamma$. 
Table 3

\begin{tabular}{lllllllll}
\hline$\gamma$ & $I(\gamma) / \gamma$ & $e(\gamma)$ & $\gamma$ & $I(\gamma) / \gamma$ & $e(\gamma)$ & \multicolumn{1}{c}{$c(\gamma) / \gamma$} & \multicolumn{1}{c}{$c$} \\
\hline 1 & 1.000 & 1.000 & 6 & 0.535 & 1.658 & 15 & 0.508 & 1.663 \\
2 & 0.690 & 1.414 & 7 & 0.527 & 1.663 & 20 & 0.505 & 1.660 \\
3 & 0.604 & 1.562 & 8 & 0.522 & 1.665 & 25 & 0.503 & 1.658 \\
4 & 0.567 & 1.620 & 9 & 0.518 & 1.666 & 30 & 0.502 & 1.656 \\
5 & 0.547 & 1.646 & 10 & 0.515 & 1.666 & $\infty$ & $1 / 2$ & $\sqrt{\mathrm{e}}$ \\
\hline
\end{tabular}

Figures 1 (a) and (b) and Table 3 show the variation of the functions $\mathrm{I}(\gamma) / \gamma$ and $\mathrm{e}(\gamma)$. We see that for an anisotropy $\gamma$ larger than 5, the difference for $\mathrm{I}(\gamma) / \gamma$ and $\mathrm{e}(\gamma)$ as compared with an infinite anisotropy is below $10 \%$ and $1 \%$, respectively.

As previously pointed out by Schilling et al. [17], we can further write the isothermal derivative

$\left.\left(\frac{\partial 4 \pi\langle M\rangle}{\partial \ln H}\right)\right|_{T}=\frac{I(\gamma)}{\gamma} \frac{\Phi_{0}}{8 \pi \lambda_{a b}^{2}(T)}$.

Knowing the asymptotic temperature dependence of $\lambda_{\mathrm{ab}}(\mathrm{T})$ for $\mathrm{T} \rightarrow \mathrm{Tc}$

$\frac{\lambda_{a b}^{2}(0)}{\lambda_{a b}^{2}(T)} \approx k\left(1-\frac{T}{T_{c}}\right)$,

with $\mathrm{k}=2$ in the BCS theory and 4 in the twofluid model [18, pp. 95, 535], allows one to estimate $\lambda_{\mathrm{ab}}(0)$ using the slope of $\partial 4 \pi\langle\mathbf{M}(\mathbf{T})\rangle / \partial \ln H$ at Tc:

$\left.\frac{\partial}{\partial T}\left(\frac{\partial 4 \pi\langle M\rangle}{\partial \ln H}\right)\right|_{T_{\mathrm{c}}}=-\frac{I(\gamma)}{\gamma} \frac{\Phi_{0}}{8 \pi \lambda_{a b}^{2}(0)} k \frac{1}{T_{\mathrm{c}}}$.

The following remarks can be made.

(1) If the samples contain impurity phases, extended defects, etc., $M^{\text {measured }}$ should be replaced by $M^{\text {measured }} / f_{S}$ where fs is the fraction of superconducting volume. In the following fits and parameter determinations, we used fs $=1$. We justify this choice by the high quality of the samples where no significant impurities were detected.

(2) As mentioned in the introduction, Hao and Clem [1,2] give an implicit solution similar to the original London model:
$-4 \pi M(T, h)=\alpha(h) \frac{\Phi_{0}}{8 \pi \lambda^{2}(T)} \ln \left(\frac{\beta(h)}{h}\right)$,

where $\mathrm{h}=H / H_{C 2}(T)$, but $\alpha$ and $\beta$ are functions of $\mathrm{H} / \mathrm{H}_{\mathrm{C} 2}(\mathrm{~T})$. In the intermediate field range 0.02 $\leq H / H_{C 2} \leq 0.3$, these two coefficients are nearly constant $(\alpha=0.77, \beta=1.44)$. This means that if we exclude the $M(T, H)$ measurements down to a few degrees below $T c(T c-5 \mathrm{~K})$, we should be in a temperature domain where $\mathrm{M} \propto \ln (\mathrm{H})$. In the following discussion, we do not take into account the field-dependent $\alpha$ and $\beta$ coefficients or equivalently, we put $\alpha=1$ and $\beta=1(\eta / \mathrm{e}=1)$. By including the Hao-Clem coefficients, the quality of the fits does not change and only $\lambda_{\mathrm{ab}}(0)$ and $\mathrm{H}_{\mathrm{C} 2, \mathrm{C}}(0)$ become approximately equal to $\sqrt{\alpha} \lambda_{\mathrm{ab}}(0)$ and $\mathrm{H}_{\mathrm{C} 2, \mathrm{C}}(0) / \beta$ as compared with the values given in Tables $4 a$ and $b, 5 a$ and $b$.

\subsection{Temperature dependence for $\mathrm{H}_{C 2}(T)$ and $\lambda(T)$, WHH model}

The true temperature dependence of the superconducting parameters such as $\lambda(\mathrm{T})$, $\mathrm{H}_{\mathrm{C} 2}(\mathrm{~T}) \ldots$ in oxide superconductors remains unsettled [16,17,19-24]. In the following, we use the WHH model [25] which gives a temperature dependence for $\xi(T)$ in between BCS and twofluid models and seems to be a reasonable approximation.

As the critical thermodynamic field follows a temperature dependence $\mathrm{H}_{\mathrm{C}}{ }^{2}(\mathrm{t}) \approx(1$ $\left.\mathrm{t}^{2}\right)^{2}, \mathrm{t}=T / T c[18, \mathrm{p} .289]$, we use for $\mathrm{H}_{\mathrm{C} 2}(\mathrm{~T})$ the approximate relation

$$
\begin{aligned}
& \mathrm{H}_{\mathrm{C} 2}(\mathrm{t})=\mathrm{H}_{\mathrm{C} 2}(0)\left(1-\mathrm{t}^{2}\right)\left(1-0.3 \mathrm{t}^{2}\right), \\
& \mathrm{H}_{\mathrm{C} 2}^{\prime}(\mathrm{Tc})=\partial H_{C 2} / \partial T / T_{c} \approx-1.4 \mathrm{H}_{\mathrm{C} 2}(0) / \mathrm{T}_{\mathrm{C}}
\end{aligned}
$$


Note that the exact solution is $\mathrm{H}^{\prime}{ }_{\mathrm{C} 2}(\mathrm{Tc})=-1.44 \mathrm{H}_{\mathrm{C} 2}(0) / \mathrm{T}_{\mathrm{C}}$ and $-1.37 \mathrm{H}_{\mathrm{C} 2}(0) / \mathrm{T}_{\mathrm{C}}$ for the dirty and clean limit, respectively.

For $\lambda^{2}(\mathrm{~T}) \propto \mathrm{H}_{\mathrm{C} 2}(\mathrm{~T}) / \mathrm{H}^{2}(\mathrm{~T})$, we have

$\lambda^{2}(t)=\lambda^{2}(0) \frac{\left(1-0.3 t^{2}\right)}{\left(1-t^{2}\right)}$ and for the G-L parameter

$\kappa(t)=\frac{\lambda(t)}{\xi(t)}=\kappa(0)\left(1-0.3 t^{2}\right)$.

Near Tc, $\lambda^{-2}(t) \approx k \lambda^{-2}(0)(1-t)$ with $\mathrm{k}=2.86$, a value between the asymptotic BCS relation $(\mathrm{k}=2)$ and that of the two-fluid model $(\mathrm{k}=4)$.

Table 4a

Eq. ( $3 \mathrm{~b}), f^{s}=1 ; \eta / e=1$ are used

\begin{tabular}{lllllll}
\hline Phase & $\gamma$ & $\begin{array}{l}\partial /\left.\partial T(\partial M / \partial \ln (H))\right|_{T^{*}} \\
\left(\mathrm{emu} / \mathrm{cm}^{3} \mathrm{~K}\right)\end{array}$ & $\begin{array}{l}T_{\mathrm{c}}^{\text {onser }}(\mathrm{K}) \\
\text { table 2 }\end{array}$ & $\begin{array}{l}\lambda_{a b}(0)(\AA) \\
k=2, \text { BCS }\end{array}$ & $\begin{array}{l}\lambda_{a b}(0)(\AA) \\
k=2.86, \text { WHH }\end{array}$ & $\begin{array}{l}\lambda_{a b}(0)(\AA) \\
k=4, \text { two fluid }\end{array}$ \\
\hline Y-123a & $5-6$ & $-5.10 \times 10^{-2}$ & 92.3 & 1210 & 1450 & 1720 \\
Y-123b & $5-6$ & $-5.83 \times 10^{-2}$ & 91.5 & 1140 & 1360 & 1610 \\
Y-124 & $5-6$ & $-3.18 \times 10^{-2}$ & 80.1 & 1650 & 1970 & 2330 \\
Y-247a & $5-6$ & $-2.82 \times 10^{-2}$ & 94.2 & 1620 & 1930 & 2290 \\
Y-247b & $5-6$ & $-3.02 \times 10^{-2}$ & 94.8 & 1560 & 1860 & 2200 \\
\hline
\end{tabular}

Table 4b

Eq. ( $3 \mathrm{~b}), f^{\prime \prime}=1 ; \eta / e=1$ are used

\begin{tabular}{lllllll}
\hline Phase & $\gamma$ & $\begin{array}{l}\partial /\left.\partial T(\partial M / \partial \ln (H))\right|_{T^{*}} \\
\left(\mathrm{emu} / \mathrm{cm}^{3} \mathrm{~K}\right)\end{array}$ & $\begin{array}{l}T_{\mathrm{c}}^{\text {onset }}(\mathrm{K}) \\
\text { table 2 }\end{array}$ & $\begin{array}{l}\lambda_{a b}(0)(\AA) \\
k=2, \text { BCS }\end{array}$ & $\begin{array}{l}\lambda_{a b}(0)(\AA) \\
k=2.86, \text { WHH }\end{array}$ & $\begin{array}{l}\lambda_{a b}(0)(\AA) \\
k=4, \text { two fluid }\end{array}$ \\
\hline Bi-2212a & $\infty$ & $-1.30 \times 10^{-2}$ & 93.7 & 2320 & 2770 & 3280 \\
Bi-2212b & $\infty$ & $-1.63 \times 10^{-2}$ & 92.4 & 2090 & 2490 & 2950 \\
T1-2201a & $\infty$ & $-1.23 \times 10^{-2}$ & 92.1 & 2400 & 2880 & 3400 \\
T1-2201b & $\infty$ & $-1.56 \times 10^{-2}$ & 92.1 & 2130 & 2550 & 3020 \\
T1-2212 & $\infty$ & $-2.23 \times 10^{-2}$ & 108.7 & 1640 & 1970 & 2320 \\
\hline
\end{tabular}

Table 5a

Fitting procedure, $f^{s}=1 ; \eta / e=1$ (see section 6.1)

\begin{tabular}{|c|c|c|c|c|c|c|c|c|c|}
\hline Phase & $\gamma$ & $\begin{array}{l}\lambda_{a b}(0) \\
(\AA)\end{array}$ & $\begin{array}{l}H_{\mathrm{c} 2, c}(0) \\
\left(10^{4} \mathrm{Oe}\right)\end{array}$ & $\begin{array}{l}T_{\mathrm{c}}^{\mathrm{L}} \\
(\mathrm{K})\end{array}$ & $\begin{array}{l}\partial H_{\mathrm{c} 2, c} /\left.\partial T\right|_{T_{\mathrm{c}}} \\
\left(10^{4} \mathrm{Oe} / \mathrm{k}\right)\end{array}$ & $\begin{array}{l}H_{\mathrm{c}}(0) \\
\left(10^{4} \mathrm{Oe}\right)\end{array}$ & $\kappa$ & $\begin{array}{l}H_{\mathrm{cl}, c}(0) \\
(\mathrm{Oe})\end{array}$ & $\begin{array}{l}\xi_{a b}(0) \\
(\AA)\end{array}$ \\
\hline$Y-123 a$ & $5-6$ & 1730 & 275 & 93.6 & -4.12 & 1.231 & 158 & 279 & 10.9 \\
\hline$Y-123 b$ & $5-6$ & 1600 & 290 & 90.4 & -4.50 & 1.364 & 151 & 321 & 10.6 \\
\hline$Y-124$ & $5-6$ & 2160 & 89 & 81.5 & -1.52 & 0.558 & 112 & 166 & 19.3 \\
\hline$Y-247 a$ & $5-6$ & 1850 & 114 & 91.5 & -1.76 & 0.742 & 109 & 225 & 16.9 \\
\hline$Y-247 b$ & $5-6$ & 2030 & 127 & 93.6 & -1.89 & 0.711 & 126 & 193 & 16.1 \\
\hline
\end{tabular}

Table 5b

Fitting procedure, $f^{\mathrm{s}}=1 ; \eta / e=1($ see section 6.2$)$ a

\begin{tabular}{|c|c|c|c|c|c|c|c|c|c|c|}
\hline Phase & $\gamma$ & $\begin{array}{l}\lambda_{a b}(0) \\
(\AA)\end{array}$ & $\begin{array}{l}H_{\mathrm{e} 2, c}(0) \\
\left(10^{4} \mathrm{Oe}\right)\end{array}$ & $\begin{array}{l}T_{\mathrm{c}}^{\mathrm{L}+\mathrm{fl}} \\
(\mathrm{K})\end{array}$ & $\begin{array}{l}\partial H_{\mathrm{c} 2, c} /\left.\partial T\right|_{T_{\mathrm{c}}} \\
\left(10^{4} \mathrm{Oe} / \mathrm{K}\right)\end{array}$ & $\begin{array}{l}H_{\mathrm{c}}(0) \\
\left(10^{4} \mathrm{Oe}\right)\end{array}$ & $\kappa$ & $\begin{array}{l}H_{\mathrm{cl}, c}(0) \\
(\mathrm{Oe})\end{array}$ & $\begin{array}{l}\xi_{a b}(0) \\
(\AA)\end{array}$ & $\alpha_{\mathrm{B}}$ \\
\hline $\mathrm{Bi}-2212 \mathrm{a}$ & $\infty$ & 2150 & 58.3 & 99.1 & -0.82 & 0.455 & 91 & 160 & 23.8 & 1.73 \\
\hline $\mathrm{Bi}-2212 \mathrm{~b}$ & $\infty$ & 2210 & 67.9 & 99.7 & -0.95 & 0.479 & 100 & 156 & 22.0 & 1.51 \\
\hline Tl-2201a & $\infty$ & 2630 & 43.2 & 94.3 & -0.64 & 0.320 & 95 & 108 & 27.6 & 1.38 \\
\hline T1-2201b & $\infty$ & 2300 & 46.7 & 99.8 & -0.66 & 0.382 & 87 & 139 & 26.5 & 1.67 \\
\hline Tl-2212 & $\infty$ & 1910 & 79.7 & 112.6 & -0.99 & 0.601 & 94 & 206 & 20.3 & 1.56 \\
\hline
\end{tabular}

We had $\left(\phi_{0} / 4 \pi \lambda^{2}\right) H_{\mathrm{c} 2}=H_{\mathrm{c}}^{2}, H_{\mathrm{c} 2}=H_{\mathrm{c}} \sqrt{2} \kappa, H_{\mathrm{c} 1} \approx \phi_{0} \ln (\kappa) / 4 \pi \lambda^{2}, H_{\mathrm{c} 2, c}(0)=\phi_{0} / 2 \pi \xi_{a b}^{2}(0)[18$, section 6.6]. 


\section{Scaling relations, fluctuation effects}

Compared to a mean-field description, the physical measurements around the critical temperature display an unusual behaviour especially for the very anisotropic phases like the bismuth and thallium compounds. In the case of the magnetization measurements, $\mathrm{M}(\mathrm{T}, \mathrm{H})$ shows an increase of the diamagnetic contribution with the external magnetic field below the mean-field critical temperature with a crossing point $\left(\mathrm{T}^{*} ; \mathrm{M}^{*}\right)$, where $\partial M /\left.\partial H\right|_{\mathrm{T}^{*}}=0$ [4,26-35]. These unexpected facts can be understood by the multilayer character and the small coherence lengths enhancing fluctuation effects [24,36-38]. Without taking into account the fluctuations, the behaviour of some parameters such as the penetration depth $\lambda(\mathrm{T})$ is unphysical. For example, $\lambda(\mathrm{T})$ apparently diverges at a temperature $\mathrm{T}^{*}$ below the mean field superconducting transition.

Recently, Bulaevskii et al. [3] have proposed to include an additional term to the free energy which takes into account the thermal fluctuations of vortices (positional fluctuation of vortices; these new excitations are called "vortons"). The expression for the fluctuation magnetization along the $\mathrm{c}$-axis $(\mathrm{H} / / \mathrm{c})$ is

$4 \pi M^{\mathrm{n}}=4 \pi \frac{k_{\mathrm{B}} T}{\phi_{0} s} \ln \left(\frac{16 \pi k_{\mathrm{B}} T \kappa^{2}}{\alpha_{\mathrm{B}} \phi_{0} s H \sqrt{\mathrm{e}}}\right)$,

with

$\kappa^{2}=\frac{\lambda_{a b}^{2}}{\xi_{a b}^{2}}=\frac{2 \pi H_{\mathrm{c} 2, c} \lambda_{a b}^{2}}{\phi_{0}}$,

where $s$ is the interlayer distance between planes or groups of superconducting planes and $\alpha_{B}$ is of order unity. This model predicts a crossing point $\left(\mathrm{T}^{*} ; \mathrm{M}^{*}\right)$ where $\mathrm{M}$ is field independent at $\mathrm{T}^{*}$,

$M\left(T^{*}\right)=-\frac{k_{\mathrm{B}} T^{*}}{\phi_{0} s} \ln \left(\frac{\eta}{e} \alpha_{\mathrm{B}} \sqrt{\mathrm{e}}\right)$,

with

$\ln \left(\frac{\eta \alpha_{\mathrm{B}}}{\sqrt{\mathrm{e}}}\right) \approx 1$

according to ref. [8] but see the remark below concerning the latter reference.

The Josephson interlayer-coupling regime is realized in bismuth and thallium compounds practically at all temperatures below Tc and only below $75 \mathrm{~K}$ in the Y-123 phase
$[3,39]$. As a consequence, it is difficult to include this term for the $\mathrm{YBaCuO}$ phases because of the small reversible domain below $T c$. The consideration of the fluctuation term within the London approach does not affect the logarithmic field dependence of the isothermal magnetization.

For a ceramic with extreme anisotropy and assuming that each grain sees the projection of $\mathrm{H}$ along the c-axis, we obtain as in section 4.1

$4 \pi\left\langle M^{\mathrm{fl}}(T, H)\right\rangle$

$$
=\frac{1}{2} 4 \pi \frac{k_{\mathrm{B}} T}{\phi_{0} s} \ln \left(\sqrt{\mathrm{e}} \frac{16 \pi k_{\mathrm{B}} T \kappa^{2}}{\alpha_{\mathrm{B}} \phi_{0} s H \sqrt{\mathrm{e}}}\right) .
$$

$\frac{\partial}{\partial T}\left(\frac{\partial 4 \pi\left\langle M^{\mathrm{n}}\right\rangle}{\partial \ln H}\right)=-\frac{1}{2} 4 \pi \frac{k_{\mathrm{B}}}{\phi_{0} s}$.

As compared to a single crystal with $\mathrm{H} / / \mathrm{c}$, the crossing-point temperature does not change but the measured magnetization $\left(\mathrm{M}\left(\mathrm{T}^{*}\right)\right)$ is reduced by a factor of two. We include the contribution of Eq. 9 (c) in the analysis of the two-dimensional $\mathrm{Bi}$ - and $\mathrm{Tl}$ phases.

Tesanovic et al. [8] have worked out a model of amplitude fluctuations of the order parameter for quasi two-dimensional superconductors near Tc. Their high-field approach gives explicitly the approximate response functions near the $\mathrm{H}_{\mathrm{C} 2}(\mathrm{~T})$ line. A scaling functional law $M /(T H)^{1 / 2}=\mathrm{f}([T-T c(H)]$ / $\left.(\mathrm{TH})^{1 / 2}\right)$ is found and has been observed by $\mathrm{Li}$ et al. [31-33] in Bi-2223 and Bi-2212 crystals. The magnetization reads

$$
\begin{aligned}
& 4 \pi M^{\mathrm{n}}(T, H) \\
& =4 \pi \frac{A k_{\mathrm{B}} \sqrt{H T}}{s \phi_{0} H_{\mathrm{c} 2}^{\prime}}\left(A \tau-\sqrt{A^{2} \tau^{2}+2}\right),
\end{aligned}
$$

with

$\tau=\frac{T-T_{c}(H)}{\sqrt{T H}}$,

$\mathrm{A}$ is a constant.

Following the authors [8], the model predicts the crossing point $\mathrm{M}^{*}\left(\mathrm{~T}^{*}\right)=k_{B} T / \phi_{o} S$ but if we derive numerically the given free energy, the magnetization obtained is in disagreement with their formula.

In the nearly three-dimensional phase Y-123, Welp et al. [40] have used a 3D identical scaling relation $M /(T H)^{2 / 3}=\mathrm{f}\left([T-T c(H)] /(T H)^{2 / 3}\right)$ near 

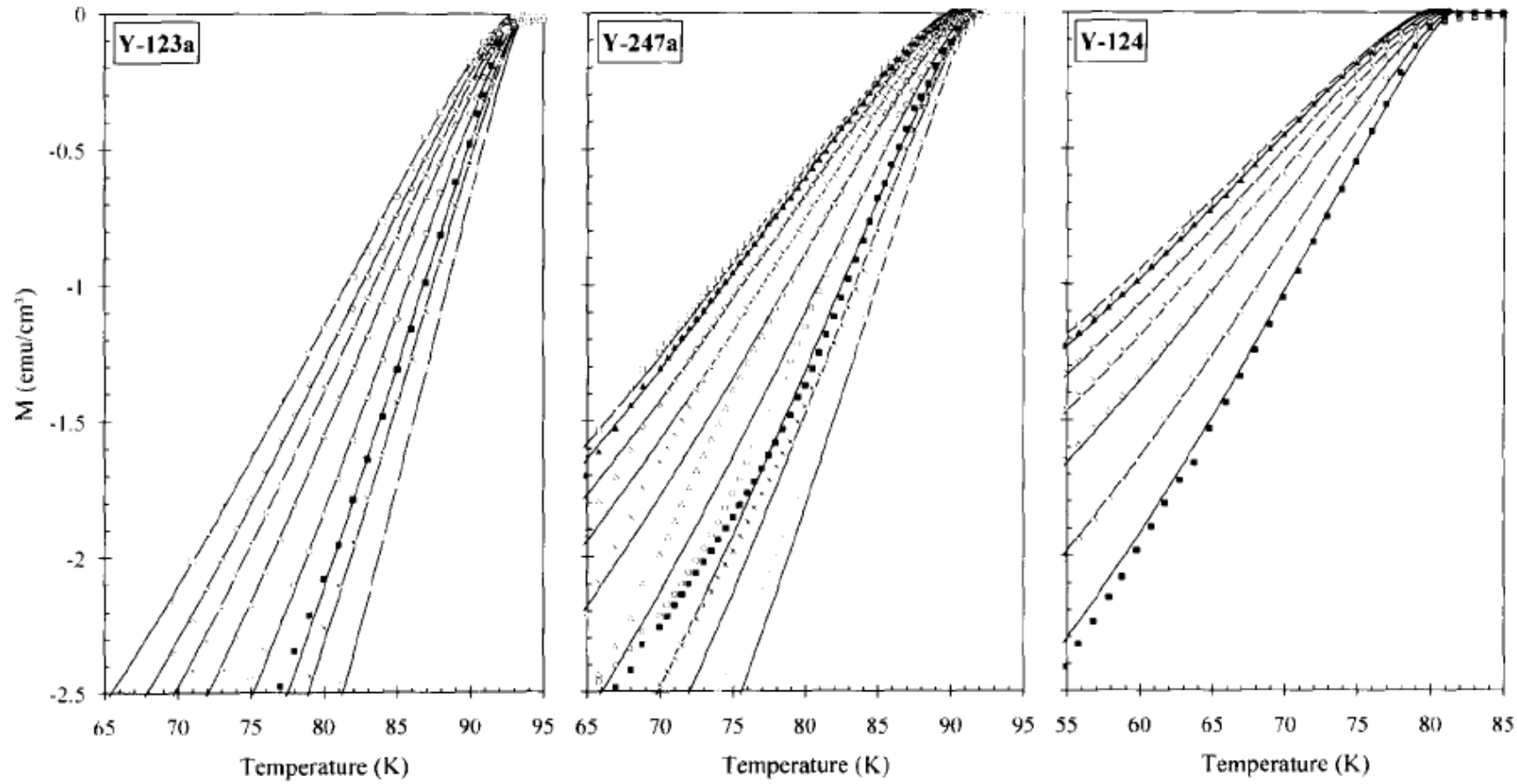

Fig. 2. Magnetization vs. temperature for different external magnetic fields and the fit (lines). The symbols $(+),(*),(\square),(O),(\Delta)$, $(\times),(\leftrightarrow),(\Delta)$ and $(\square)$ correspond to $1,3,5,10,20,30,40,50$ and $55 \mathrm{kOe}$, respectively.

the $\mathrm{H}_{\mathrm{C} 2}(\mathrm{~T})$ line (high-field result). The $M(T, H)$ functional is not given explicitly.

Below Tc, Cho et al. [23] have observed in a Bi2212 single crystal surprising scalings $M(T, H)$ / $\mathrm{M}\left(\mathrm{T}^{\prime}, \mathrm{H}\right)$ versus $\mathrm{T}$ and $M(T, H) / M\left(T, H^{\prime}\right)$ versus $\mathrm{H}$ where $\left(\mathrm{T}^{\prime}, \mathrm{H}^{\prime}\right)$ is an arbitrary point in the reversible domain. They conclude that $\mathrm{M}(\mathrm{T}, \mathrm{H})$ can be written as $M(T, H)=f(T) g(H)$ and find that $\mathrm{H}_{\mathrm{C} 2}$ is quasi temperature independent in this temperature range.

\section{Parameter determination, fitting procedure and scaling}

\subsection{YBaCuO phases}

Table 4a summarizes the temperature derivatives of the measured logarithmic slopes $\partial M(T) / \partial \ln H$ and values of $\lambda_{\mathrm{ab}}(0)$ directly obtained from Eq. (3b) with three different temperature dependences near Tc. We adopted an anisotropy parameter $\gamma=5$ to 6 for all three compounds, in agreement with previous results $[22,35,38,41]$.

The analysis presented in Table $5 \mathrm{a}$ is based on a fit of the $\mathrm{M}(\mathrm{T}, \mathrm{H})$ data in the reversible regime but omitting the region near Tc, i.e. $\mathrm{T}<\mathrm{Tc}-5 \mathrm{~K}, \mathrm{H} \geq 5 \mathrm{kOe}$. The free parameters entering Eq. (3a) (London model without vortex fluctuations) are $\lambda_{\mathrm{ab}}(0), \mathrm{H}_{\mathrm{C} 2}(0)$ and $\mathrm{Tc}$ labeled $\mathrm{T}_{\mathrm{C}}^{\mathrm{L}}$, under the assumption of a WHH temperature dependence of $\lambda$ and $\mathrm{H}_{\mathrm{C} 2}$. The data shown in Figs. 2 to 4 are completed by the fitted $\mathrm{M}(\mathrm{T}, \mathrm{H})$ curves in Fig. 2.

\subsection{BiSrCaCuO and TIBaCaCuO phases}

Again, $\lambda_{\mathrm{ab}}(0)$ was first determined from the slope of $\partial \mathrm{M}(\mathrm{T}) / \partial \mathrm{InH}$ versus $\mathrm{T}$. Ignoring the fluctuation term formally results in a divergence of the penetration depth at $\mathrm{T}^{*}$ but the difference of $\partial / \partial \mathrm{T}(\partial \mathrm{M} / \partial \mathrm{lnH})$ and $\lambda_{\mathrm{ab}}(0)$ is estimated not to exceed $4 \%$ and $2 \%$, respectively. Considering the more important uncertainty concerning the appropriate value of $\mathrm{k}$, we indicate in Table $4 \mathrm{~b}$ values according to Eq. (3b).

Secondly, we have tested a fit similar to that used for the $\mathrm{YBaCuO}$ phases but with an infinite anisotropy [30,35] (i.e. $\mathrm{I}(\gamma) / \gamma=1 / 2, \mathrm{e}(\gamma)=\sqrt{\mathrm{e}}$ ). However, the fit on the basis of the original London model is clearly unsatisfactory. We have therefore analyzed our reversible $\mathrm{M}(\mathrm{T}, \mathrm{H})$ data ( $\mathrm{T}<\mathrm{Tc}, \mathrm{H} \geq 10 \mathrm{kOe}$ ) using Eqs. (3a) and (9c) and a WHH temperature dependence for $\lambda$, $\mathrm{H}_{\mathrm{C} 2}$ and $\kappa$. The effective interlayer parameter $\mathrm{s}^{\mathrm{C}}$ is determined from the observed crossing point 

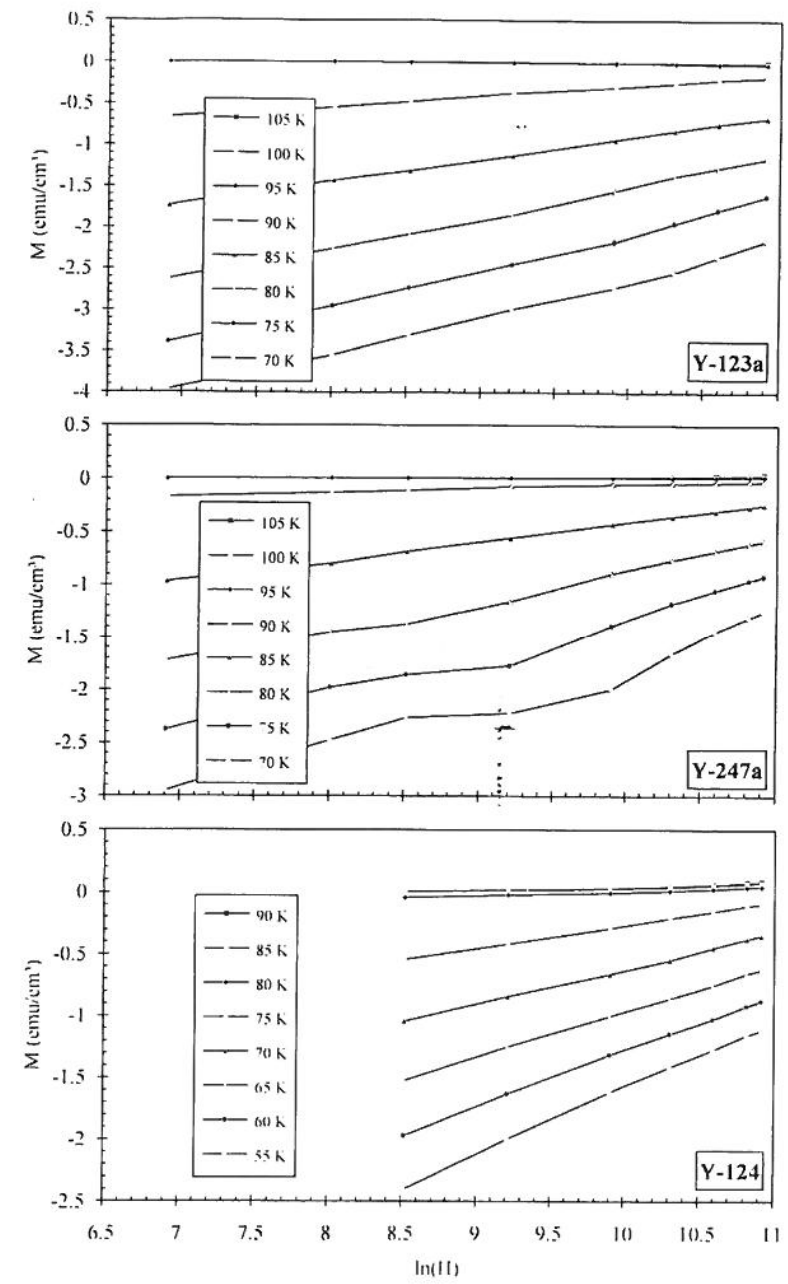

Fig.3. Isothermal magnetization vs. $\ln (\mathrm{H} /$ Oersted).

$\left(\mathrm{T}^{*} ;\left(M\left(T^{*}\right)\right)\right)($ Table 6$)$. We note that the values of $s$ determined in this way are always larger than those corresponding to the crystal structure $\left(\mathrm{s}^{\mathrm{C}}\right.$, see Table 2). The discrepancy is likely to result from the assumed $100 \%$ superconducting volume or from $\eta \alpha_{\mathrm{B}} / \sqrt{\mathrm{e}}$ being different from 1 . The free parameters are $\lambda_{\mathrm{ab}}(0), \mathrm{H}_{\mathrm{C} 2, \mathrm{C}}(0), \alpha_{\mathrm{B}}$ and Tc labeled here $\mathrm{T}_{\mathrm{C}}{ }^{\mathrm{L}+\mathrm{fl}}$ (Tables $5 \mathrm{~b}$ and 6 ).

Table 6

We made use of $s=-k_{\mathrm{B}} T^{*} / \phi_{0} 2\left\langle M\left(T^{*}\right)\right\rangle$

\begin{tabular}{llcc}
\hline Phase & $M^{*}\left(\mathrm{emu} / \mathrm{cm}^{3}\right)$ & $T^{*}(\mathrm{~K})$ & $s(\AA)$ \\
\hline Bi-2212a & -0.125 & 92.4 & 24.6 \\
Bi-2212b & -0.145 & 90.5 & 20.8 \\
Tl-2201a & -0.064 & 88.8 & 46.3 \\
Tl-2201b & -0.173 & 88.8 & 17.1 \\
Tl-2212 & -0.145 & 105.0 & 24.6 \\
\hline
\end{tabular}

Figs. 5, 6, 7 and 8 show the $\mathrm{M}(\mathrm{T}, \mathrm{H})$ data and the fit, a zoom near $\mathrm{T}^{*}$, the magnetization versus $\operatorname{In}(\mathrm{H})$ and the $\partial M / \partial \ln H$ versus temperature for the Bi-2212, T1-2201 and T12212 phases.

Concerning the quality of the fits, let us mention that $\chi^{2}$ amounts to about $2.5 \%$ for the $\mathrm{Bi}$ and $\mathrm{T} 1$ compounds, compared to $1.5 \%$ for the $\mathrm{YBaCuO}$ phases. Without the inclusion of a vortex-fluctuation term in the former case, $\chi^{2}$ would increase to typically $15 \%$ in the appropriate temperature and field ranges.

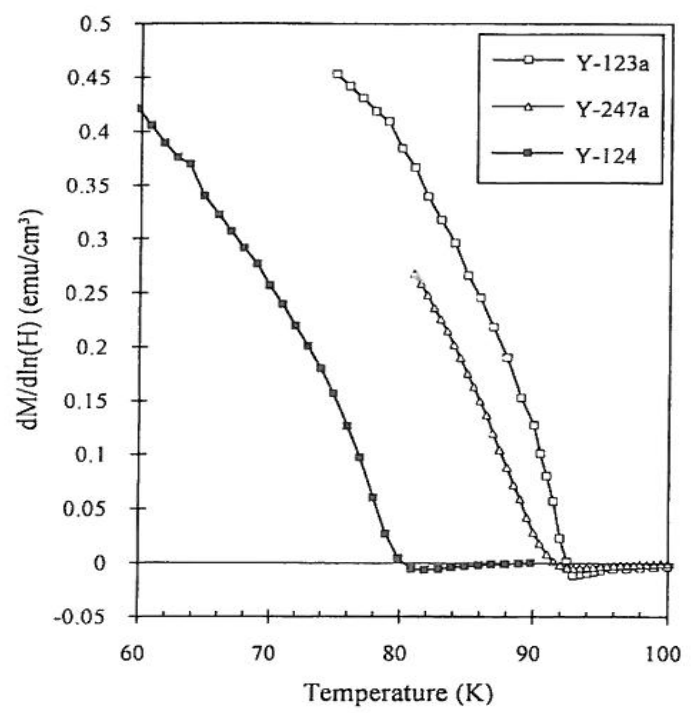

Fig. 4. $\partial M / \partial \ln H$ vs. temperature calculated usually between $B=1$ to $5 \mathrm{~T}$. These curves reflect the variation of $H_{\mathrm{cl}} / \ln (\kappa)$ with $T$.

\subsection{Scalings}

For the two-dimensional phases, we have tried to introduce the scaling proposed by Tesanovic el al. [8]. The rather unsuccessful test may indicate that the polycrystalline nature of ceramics is not suitable in this respect. However, following Cho et al. [23], we also observe the same scalings $M(T, H) / M\left(T^{\prime}, \mathrm{H}\right)$ versus $\mathrm{T}$ and $M(T, H) / M\left(T, H^{\prime}\right)$ versus $\mathrm{H}$, below $\mathrm{Tc}-10 \mathrm{~K}$ for $\mathrm{BiSrCaCuO}$ and $\mathrm{T} 1 \mathrm{BaCaCuO}$ but not for the $\mathrm{YBaCuO}$ phases. As an example, we present in Figs. 9(a), (b) and (c) the scalings for the T12212 phase.

In the spirit of the surprising findings of Cho et al., we conclude from Fig. 9 that the factorization of $\mathrm{M}(\mathrm{T}, \mathrm{H})$ into a function of temperature and a function of field $(\approx \ln H)$ within the reversible region is also verified for the polycrystalline strongly two-dimensional compounds exemplified by TI-2212. 

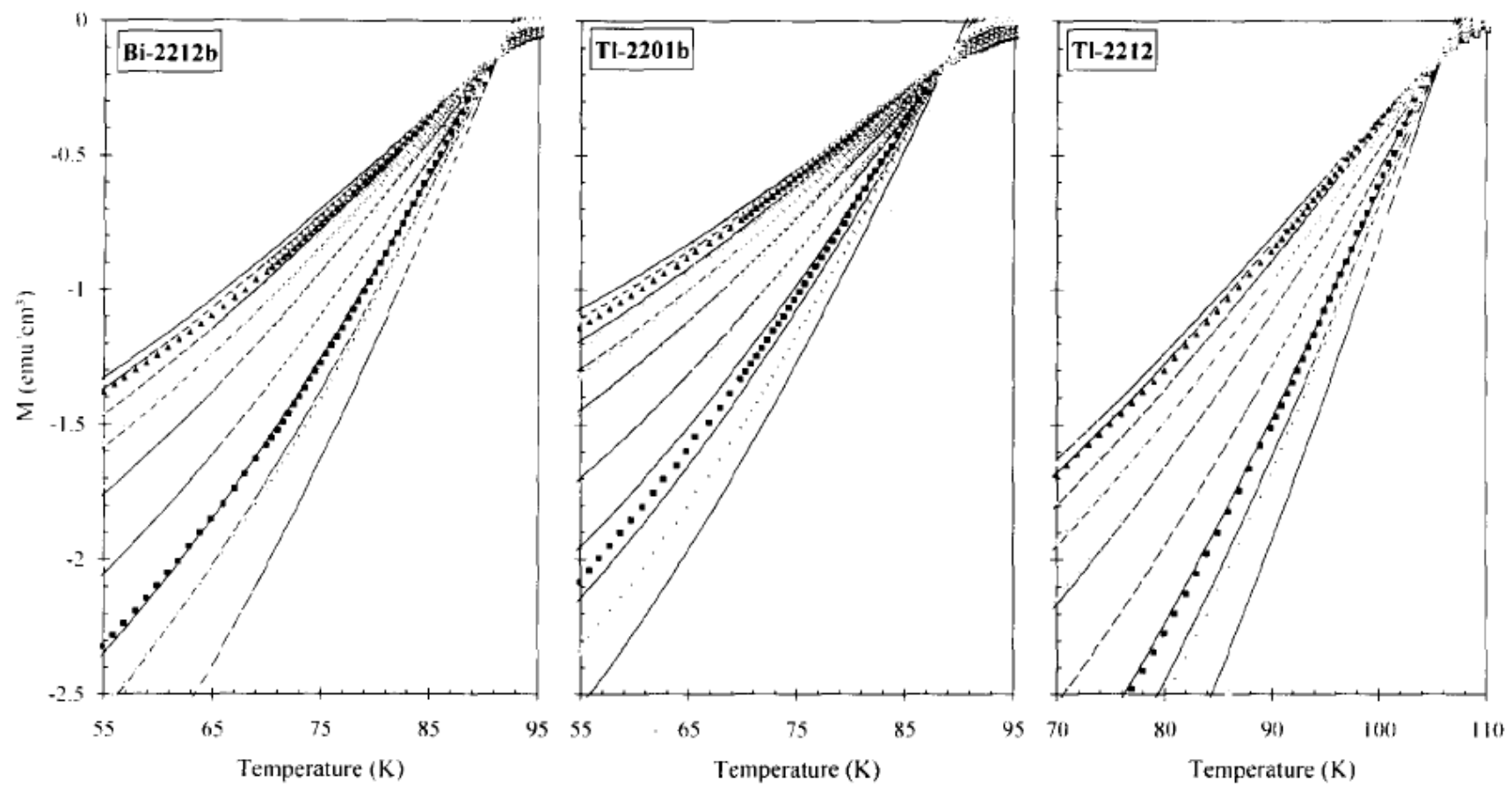

Fig. 5. Magnetization vs. temperature for different external magnetic fields and the fit (lines). The symbols $(+),(*),(\square),(O),(\Delta)$, $(X),(\leftarrow),(\Delta)$ and $(\square)$ correspond to $1,3,5,10,20,30,40,50$ and $55 \mathrm{kOe}$, respectively.
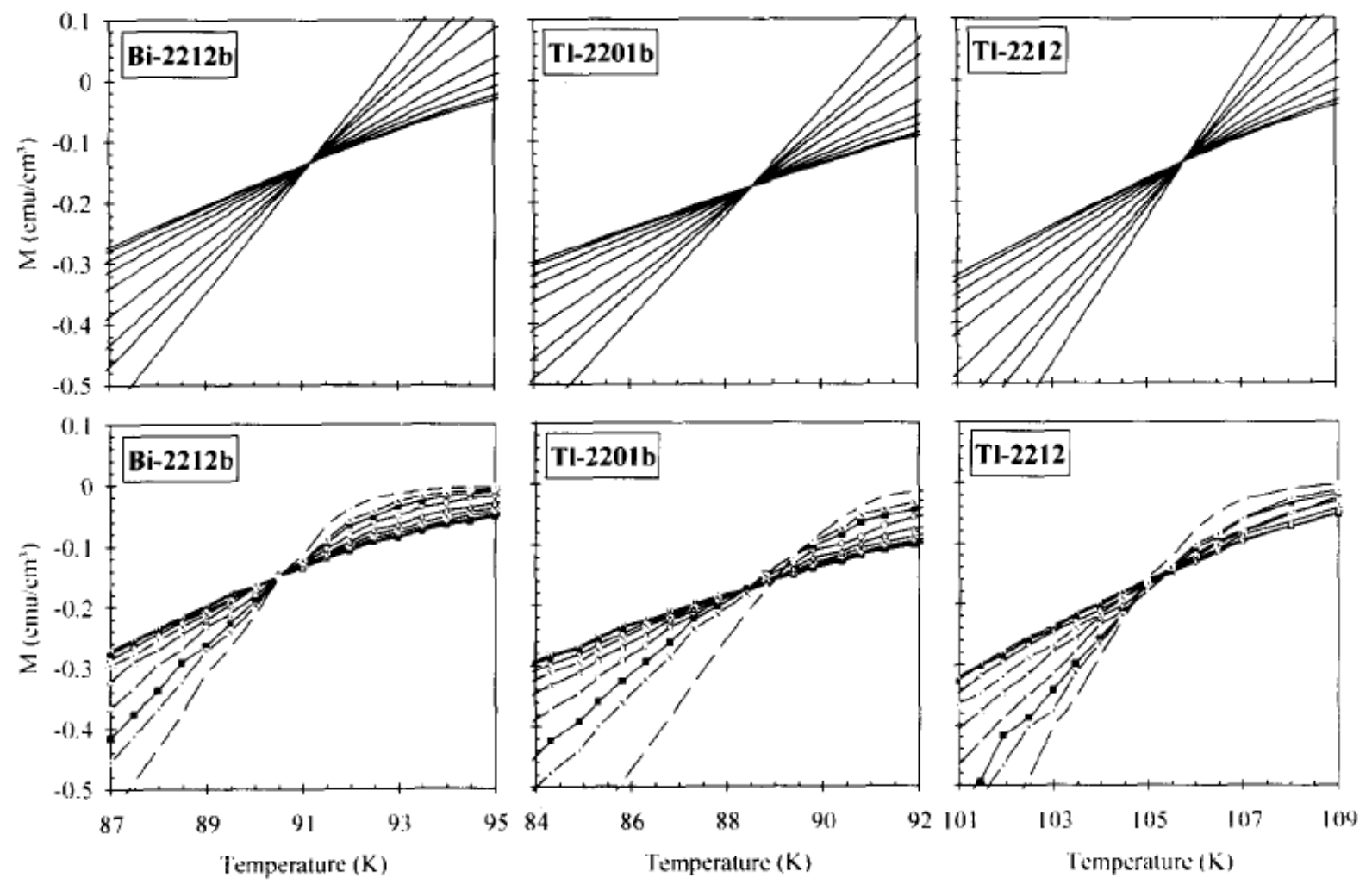

Fig. 6. Enlarged representation of Fig. 5 near $T^{*}$ (lower part; lines are guide for the eyes) and the fits with a vortex-fluctuation tern (upper part). Symbols as in Fig. 5. 

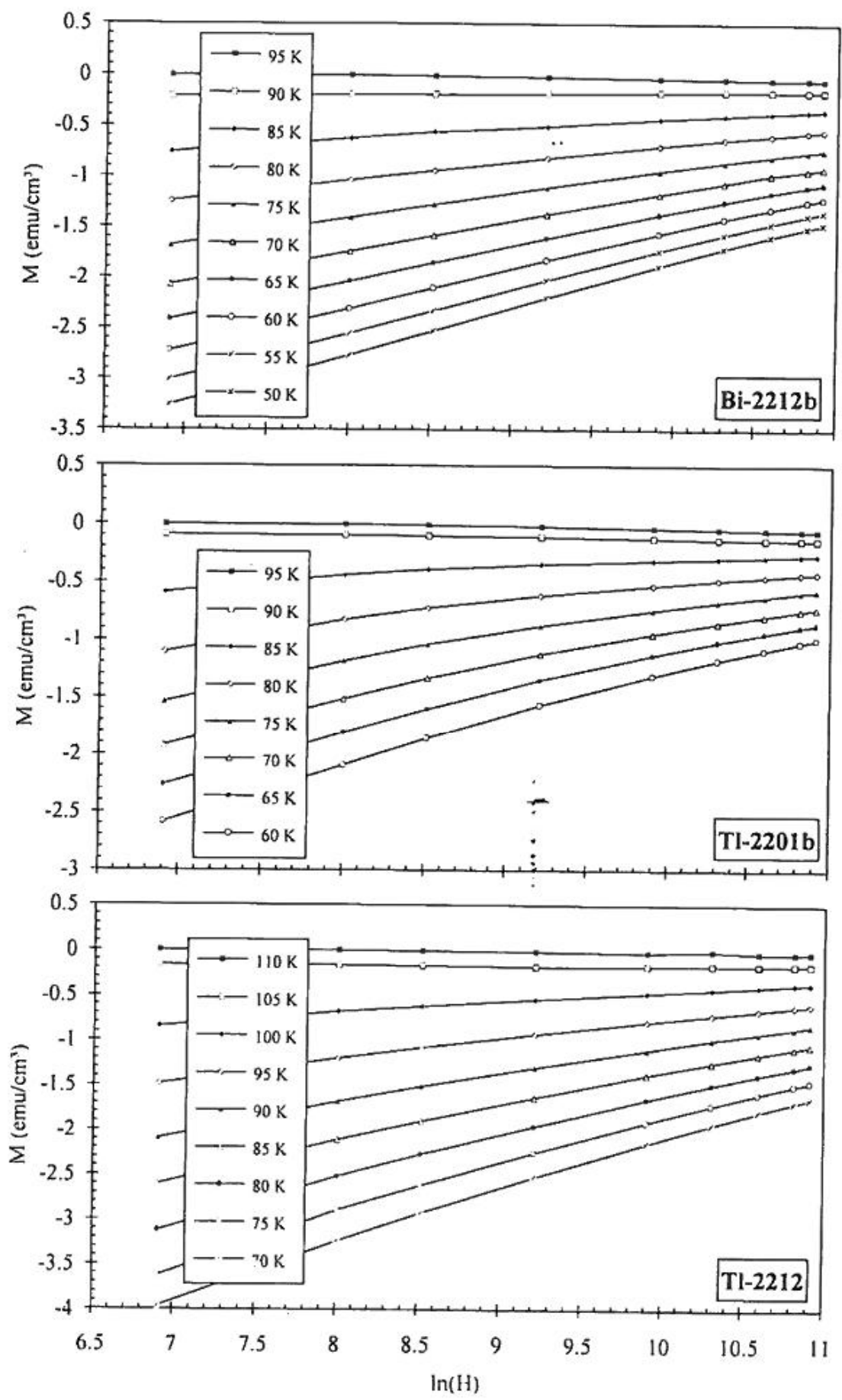

Fig. 7. Isothermal magnetization vs. $\ln (H /$ Oersted $)$. 


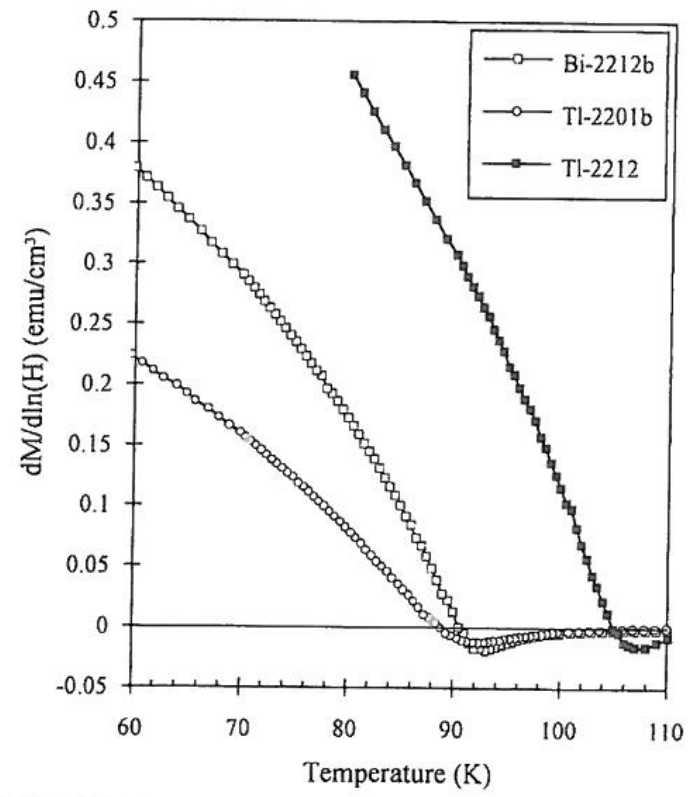

Fig. 8. $\partial M / \partial \ln H$ vs. temperature calculated usually between $B=1$ to $5 \mathrm{~T}$. These curves reflect the variation of $H_{\mathrm{c} 1} / \ln (\kappa)$ with $T$.

\section{Discussion}

\section{I. YBaCuO phases}

The magnetization in $\mathrm{YBaCuO}$ ceramics does not clearly exhibit a crossing point ( $\mathrm{T}^{*}$; $\left.\mathrm{M}^{*}\right)$. Further, in the $\partial \mathrm{M} / \partial 1 \mathrm{n} H$ versus temperature graphs (Fig. 4) the temperature where this slope vanishes practically coincides with the onset critical temperature. It appears that these phases do not require the inclusion of a substantial fluctuation term. In the reversible regime, the isothermal magnetization follows a logarithmic field dependence. The fitted critical temperatures $\mathrm{Tc}^{\mathrm{L}}$ are also comparable to the $\mathrm{Tc}^{\text {onset }}$ low-field values.

As regards Y-123: the slope of the upper critical field we determined for the Y-123 phase $(-4.3 \mathrm{~T} / \mathrm{K})$ is larger than previous results given in the literature, i.e. - $1.9 \mathrm{~T} / \mathrm{K}$ [41-43], $-2.3 \mathrm{~T} / \mathrm{K}$ [44] by extrapolation of the magnetization below Tc or - $1.9 \mathrm{~T} / \mathrm{K}$ [40], - 1.8 [38] by highfield scaling. On the other hand, the present result is closer to that of a new specific-heat analysis of a single crystal ( $-3.2 \mathrm{~T} / \mathrm{K}$ [45]) and a ceramic $(-4.5 \mathrm{~T} / \mathrm{K}[46])$. The in-plane penetration depth is found to be $\lambda_{a b}(0)=1400 \AA$ by $\mu^{+}$SR [22] or by magnetic measurements $[17,21]$. Our result is in good agreement using the slope of $\partial \mathrm{M} / \partial 1 \mathrm{n} H$ but somewhat larger using the fitting procedure (Tables $4 \mathrm{a}$ and $5 \mathrm{a}$ ).

On Y-124 we remark that for the Y-124 phase, $\left.\mu о \partial H_{c 2, \mathrm{~d}} \partial \mathrm{\partial T}\right|_{T c}=-1.6 \mathrm{~T} / \mathrm{K} \quad[47], \lambda_{\mathrm{ab}}(0)=1960-$ $2000 \AA$ [17], $\mathrm{H}_{\mathrm{C} 1}(0)=180$ Oe [17] are mentioned in the literature. Our results, - 1.5 $\mathrm{T} / \mathrm{K}, 2060 \AA$, and 166 Oe compare favorably with the published parameters.

Finally, Y-247: we have not found comparable measurements for this phase in the literature.

\subsection{BiSrCaCuO and TIBaCaCuO phases}

The magnetization in $\mathrm{BiSrCaCuO}$ and TIBaCaCuO ceramics does show a crossing point $\left(\mathrm{T}^{*} ; \mathrm{M}^{*}\right)$ below the mean-field superconducting transition, a fact illustrating the very large anisotropy of these compounds.

The fitted values of $\alpha_{B}$ are near the number $\sqrt{e}$ by using $\eta / \mathrm{e}=1$, i.e. $\ln \left(\eta \alpha_{\mathrm{B}} / \sqrt{\mathrm{e}}\right) \approx 1$ as postulated by Tesanovic et al. [8]. The fits, however, yield too large values of the meanfield critical temperature $\mathrm{Tc}^{\mathrm{L}+\mathrm{fl}}$. The isothermal magnetization deviates slightly from the logarithmic field dependence expected in the London regime including the vortex-fluctuation term. This may reflect, as mentioned by Hao and Clem [1,2], the presence of interactions between the vortices. We feel that including the fluctuation of vortices in the original London approach is not sufficient to explain the behavior of the reversible magnetization of the quasi 2D oxide superconductors. The overestimate of the critical temperature $\mathrm{Tc}^{\mathrm{L}+\mathrm{fl}}$ is possibly a characteristic feature of the vortex- fluctuation theory.

As first observed by Cho et al. [23] we also found scalings below $\mathrm{Tc}^{\text {onset }}-10 \mathrm{~K}$ for these $2 \mathrm{D}$ compounds which imply that $M(T, H)$ can be written as $f(T) g(H)$. Consequently, one expects a wide temperature region where $\mathrm{H}_{\mathrm{C} 2}$ is nearly constant, but this conclusion depends on the assumption that the scaling is really exact.

As regards $\mathrm{Bi}-2212$ : the critical field slope $\mu \circ \partial H_{c 2, c} /\left.\partial T\right|_{T c}$ at $\mathrm{Tc}$ found in the present work $(0.9 \mathrm{~T} / \mathrm{K})$ is markedly lower than values previously indicated, i.e. $1.4 \mathrm{~T} / \mathrm{K}[2,29,48]$ or even $2.7 \mathrm{~T} / \mathrm{K}$ [33]. The results for $\lambda_{\mathrm{ab}}(0)$ also vary widely: $3000 \AA$ [20], 3420-3900 ̊ [28], 
$1780 \AA$ [48] and $2100 \AA$ [49], compared to the presently estimated $2200 \AA$.

On T1-2201 we remark that a very small slope $0.65 \mathrm{~T} / \mathrm{K}$ consistently obtained for two samples appears to he characteristics for this phase. The mean value of $\lambda_{\mathrm{ab}}(0)=2400 \AA$ has to be compared with that of 1700 A obtained by Zuo et al. [35].

Finally we note on T1-2212 that our result of $1.0 \mathrm{~T} / \mathrm{K}$ from magnetization is perfectly in line with that obtained from resistive measurements [50].

\section{Conclusions}

The analysis of the reversible magnetization of high-Tc superconductors allows one to test theories and to determine important physical parameters. For the three $\mathrm{YBaCuO}$ phases, using the original London model we can fit the reversible $M(H, T)$ measurements without including any other term. The parameters of $\mathrm{Y}-247$, such as $\mathrm{H}_{\mathrm{C} 2}$ and $\lambda$, are between those of the two phases which compose its structure, i.e. Y- 123 and Y-124. For $\mathrm{BiSrCaCuO}$ and $\mathrm{TIBaCaCuO}$, extremely twodimensional phases, the inclusion of a term to take into account the thermal fluctuation of vortices appears justified but the quantitative analysis is not entirely satisfactory.

\section{Acknowledgements}

The authors are grateful to J.A. Fernandez, F. Liniger and A. Naula for their technical assistance. This work was supported by the Fonds National Suisse de la Recherche Scientifique.
Appendix

Based on ref. [16], the projection of the magnetization along the applied field $H$ is written as

$M(H, T)^{\| H}=-M_{0}(T, \theta) \sqrt{m(\theta)}, \quad \theta=[\boldsymbol{H}, \boldsymbol{c}]$.

with

$$
\begin{aligned}
& M_{0}(T, \theta)=\frac{\Phi_{0}}{32 \pi^{2} \lambda^{2}(T, \theta)} \ln \left(\frac{\eta}{\mathrm{e}} \frac{H_{\mathrm{c} 2}(T, \theta)}{H}\right), \\
& \sqrt{m(\theta)}=\sqrt{\gamma^{-2 / 3} \sin ^{2}(\theta)+\gamma^{4 / 3} \cos ^{2}(\theta)} \\
& =\frac{\sqrt{\sin ^{2}(\theta)+\gamma^{2} \cos ^{2}(\theta)}}{\gamma^{1 / 3}}=\frac{\epsilon(\theta)}{\gamma^{1 / 3}}, \\
& H_{\mathrm{c} 2}(T, \theta)=\frac{H_{\mathrm{c} 2, a b}(T)}{\sqrt{\sin ^{2}(\theta)+\gamma^{2} \cos ^{2}(\theta)}}=\frac{H_{\mathrm{c} 2, a b}(T)}{\epsilon(\theta)} \\
& =\frac{\gamma H_{\mathrm{c} 2, c}(T)}{\sqrt{\sin ^{2}(\theta)+\gamma^{2} \cos ^{2}(\theta)}}=\frac{\gamma H_{\mathrm{c} 2, c}(T)}{\epsilon(\theta)}, \\
& \lambda=\sqrt[3]{\lambda_{a b}^{2} \lambda_{c}}, \lambda=\gamma^{1 / 3} \lambda_{a b}, \lambda=\gamma^{-2 / 3} \lambda_{c} .
\end{aligned}
$$

Under the assumption that the parameter $\eta$ does not depend on $\theta$, the measured magnetization $\langle M(T$. $H)>$ for ceramics is

$$
\begin{aligned}
& \langle M(T, H)\rangle= \\
& \quad-\int_{0}^{\pi / 2} M_{0}(T, \theta) \sqrt{m(\theta)} \sin (\theta) \mathrm{d}(\theta) \\
& =-\int_{0}^{\pi / 2} \frac{\Phi_{0}}{32 \pi^{2} \lambda_{a b}^{2}(T)} \frac{1}{\gamma^{2 / 3}} \\
& \times \ln \left(\frac{\eta}{e} \frac{\gamma H_{\mathrm{c} 2, c}(T)}{\epsilon(\theta) H}\right) \frac{\epsilon(\theta)}{\gamma^{1 / 3}} \sin (\theta) \mathrm{d}(\theta) .
\end{aligned}
$$

Using the substitution $x=\cos (\theta)$, we obtain

$$
\begin{aligned}
& -4 \pi\langle M(T, H)\rangle \\
& =\frac{\Phi_{0}}{8 \pi \lambda_{a b}^{2}(T)} \frac{1}{\gamma}\left\{\ln \left(\frac{\eta}{e} \frac{\gamma H_{\mathrm{c} 2, c}(T)}{H}\right) I(\gamma)-g(\gamma)\right\}, \\
& I(\gamma)=\int_{0}^{\pi / 2} \epsilon(\theta) \sin (\theta) \mathrm{d} \theta=\int_{0}^{1} \sqrt{1+\left(\gamma^{2}-1\right) x^{2}} \mathrm{~d} x \\
& =\frac{1}{2}\left\{\gamma+\frac{1}{\sqrt{\left(\gamma^{2}-1\right)}} \ln \left(\gamma+\sqrt{\left(\gamma^{2}-1\right)}\right)\right\},
\end{aligned}
$$




$$
\begin{aligned}
g(\gamma) & =\int_{0}^{\pi / 2} \epsilon(\theta) \ln (\epsilon(\theta)) \sin (\theta) \mathrm{d} \theta \\
& =\int_{0}^{1} \sqrt{1+\left(\gamma^{2}-1\right) x^{2}} \ln \sqrt{1+\left(\gamma^{2}-1\right) x^{2}} \mathrm{~d} x .
\end{aligned}
$$

Finally

$-4 \pi\langle M(T, H)\rangle$

$$
=\frac{\Phi_{0}}{8 \pi \lambda_{a b}^{2}(T)} \frac{I(\gamma)}{\gamma} \ln \left(e(\gamma) \frac{\eta}{e} \frac{H_{\mathrm{c} 2, c}(T)}{H}\right),
$$

with

$$
e(\gamma)=\gamma \mathrm{e}^{-g(\gamma) / I(\gamma)} .
$$

\section{References}

[1] Z. Hao and J.R. Clem, Phys. Rev. Lett. 67 (1991) 2371.

[2] Z. Hao, J.R. Clem, M.W. McElfresh, L. Civale, A.P. Malozemoffand F. Holtzberg, Phys. Rev. B 43 ( 1991 ) 2844.

[3] L.N. Bulaevskii, M. Ledvij and V.G. Kogan, Phys. Rev. Lett. 68 (1992) 3773.

[4] W.J. Skocpol and M. Tinkham, Rep. Prog. Phys. 38 (1975) 1049.

[5] L.N. Bulaevskii, V.L. Ginzburg and A.A. Sobyanin, Physica C 152 (1988) 378.

[6] R. Ikeda and T. Tsuneto, J. Phys. Soc. Jpn. 60 ( 1991 ) 1337.

[7] L.I. Glazman and A.E. Koshelev, Phys. Rev. B 43 ( 1991 ) 2835.

[8]Z. Tesanovic, L. Xing, L.N. Bulaevskii, Q. Li and $\mathrm{M}$.

Suenaga, Phys. Roy. Lett. 68 (1992) 3563.

[9] M.B. Salamon, J. Shi, N. Overend and M.A. Howson, Phys.

Rev. B 47 (1993) 5520.

[10] A. Buzdin and D. Feinberg, Physica C 220 (1994) 74.

[11] D.C. Johnston, J. Mag. Mag. Mater. 100 (1991) 218.

[12] G. Triscone, J.-Y. Genoud, T. Graf and J. Muller, Physica C 176 (1991) 247.

[13] G. Triscone, A. Junod, J. Muller, C. Opagiste, M. Couach, A.F. Khoder, T.K. Jondo,
J.-L. Jorda and M.Th. Cohen-Adad, J. Alloys and Comp. 195 (1993) 607.

[14] G. Triscone, J.-Y. Genoud, T. Graf, A. Junod and J. Muller, J. Alloys and Comp. 195 (1993) 351.

[15] P.G. de Gennes, Superconductivity of Metals and Alloys (Benjamin, New York, 1966). [16]V.G. Kogan, M.M. Fang and S. Mitra, Phys. Rev. B 38 (1988) 11958.

[17] A. Schilling, F. Hulliger and H.R. Ott, Physica C 168 (1990) 272.

[18] T.P. Orlando and K.A. Delin, Foundations of Applied Superconductivity (Addison-Wesley, Reading, 1991 ).

[19] Y.R. Wang and M. Rice, Phys. Rev. B 38 (1988) 7163.

[20] S. Mitra, J.H. Cho, W.C. Lee, D.C. Johnston and V.G. Kogan, Phys. Rev. B 40 (1989) 2674.

[21] L. Krusin-Elbaum, R.L. Greene, F. Holtzber, A.P. Malozemoff and Y. Yeshurun, Phys. Rev. Lett. 62 (1989) 217.

[22]D.R. Harshman, L.F. Schneemeyer, J.V. Waszczak, G.

Aeppli, R.J. Cava, B. Batlogg, L.W. Rupp, E.J. Ansaldo and D.LI. Williams, Phys. Rev. B 39 (1989) 851.

[23] J.H. Cho, Z.-D. Hao and D.C. Johnston, Phys. Rev. B 46 (1992) 8679.

[24] V.G. Kogan, M. Ledvij, A.Yu. Simonov, J.H. Cho and D.C. Johnston, Phys. Rev. Lett. 70 (1993) 1870.

[25] N.R. Werthamer, E. Helfand and P.C. Hohenberg, Phys.

Rev. 147 (1966)295.

[26] R. Ikeda et al., J. Phys. Soc. Jpn. 58 (1989) 1377.

[27] R. Ikeda et al., J. Phys. Soc. Jpn. 59 (1990) 1397.

[28] W. Kritscha, F.M. Sauerzopf, H.W. Weber, G.W. Crabtree, Y.C. Chang and P.Z. Jiang, Physica C 179 ( 1991 ) 59.

[29] P.H. Kes, C.J. Van der Beck, M.P. Maley, M.E. McHenry,

D.A. Huse, M.J.V. Menken and A.A. Menovsky, Phys. Rev. Lett. 67 (1991) 2383.

[30] J.C. Martinez, S.H. Brongersma, A. Koshelev, B. Ivlev, P.H. Kes, R.P. Griessen, D.G. de Groot, Z. Tarnavski and A.A. 
Menovski, Phys. Rev. Left. 69 (1992) 2276.

[31] Q.-A. Li, M. Suenaga, J.-H. Gohng and D.K. Finnemore, Phys. Rev. B 46 (1992) 3195; Q.-A. Li, M. Suenaga, T. Hikata and K. Sato, Phys. Rev. B46 (1992) 5857.

[32] Q.-A. Li, K. Shibutani, M. Suenaga, I. Shigaki and R. Ogawa, in: Proc. of LT-20 (1993), Eugene USA.

[33] Q.-A. Li, IC Shibutani, M. Suenaga, I. Shigaki and R. Ogawa, Phys. Rev. B 48 (1993) 9877.

[34] C. Opagiste, M. Couach, A.F. Khoder, F. Monnier, A. Junod, G. Triscone, J. Muller, J.L. Jorda, T.K. Jondo and M.Th. Cohen-Adad, J. Alloys and Comp. 195 (1993) 455.

[35] F. Zuo, D. Vacaru, H.M. Duan and A.M. Hermann, Phys. Rev. C 47 (1993) 8327.

[36] R.A. Klemm, M.R. Beasley and A. Luther, Phys. Rev. B 8 (1973) 5072.

[37] D.R. Nelson, Phys. Rev. Lett. 60 (1988) 1973.

[38] A. Junod, E. Bonjour, R. Calemczuk, J.Y. Henry, J. Muller, G. Triscone, and J.C. Vallier, Physica C 211 (1993) 304.

[39] L.N. Bulaevskii, M.P. Maley and I.F. Schegolev, in: Proc. of LT-20 (1993), Eugene USA.

[40] U. Welp, S. Fleshier, W.K. Kwok, R.A. Klemm, V.M. Vinokur, J. Downey, B. Veal and G.W. Crabtree, Phys. Rev. Lett. 67 (1991) 3180. [41] K.G. Vandervoort, U. Welp, J.E. Kessler, H. Claus, G.W. Crabtree, W.K. Kwok, A. Umezawa, B.W. Veal, J.W. Downey, A.P. Paulikas and J.Z. Liu, Phys. Rev. Lett. 43 (1991) 13042.

[42] U. Welp, W.K. Kwok, G.W. Crabtree, K.G. Vandervoort and J.Z. Liu, Phys. Rev. Lett. 62 (1989) 1908.

[43] U. Welp, M. Grimsditch, H. You, W.K. Kwok, M.M. Fang, G.W. Crabtree and J.Z. Liu, Physica B 163 (1990) 473.
[44] J.-Y. Genoud, T. Graf, A. Junod, G. Triscone and J. Muller, Physica C 185-189 (1991) 597.

[45 ] E. Janod, to be published.

[46] A. Junod, to be published.

[47] W.C. Lee and D.M. Ginsberg, Phys. Rev. B 45 (1992) 7402.

[48] L. Zhang, J.Z. Lice and R.N. Shelton, Phys. Rev. B 45 (1992) 4978.

[49 ] A. Schilling, R. Jin, J.D. Guo and H.R. Ott, in: Proc. of LT-20 (1993), Eugene USA.

[50] J.H. Kang, K.E. Gray, R.T. Kampwirth and D.W. Day, Appl. Phys. Lett. 53 (1988) 2560.

[51] E. Janod, A. Junod, T. Graf, K.-Q. Wang, G. Triscone and J. Muller, Physica C 216 (1993) 129.

[52] A. Junod, D. Eckert, T. Graf, G. Triscone and J. Muller, Physica C 162-164 (1989)482.

[53] A. Junod, D. Eckert, T. Graf, E. Kaldis, J. Karpinski, S. Rusiecki, D. Sanchez, G. Triscone and J. Muller, Physica C 168 (1990) 47.

[54] G. Triscone, T. Graf, A. Junod, D. Sanchez, O. Brunner, D. Cattani and J. Muller, Physica C 168 (1990) 40.

[55] J.-Y. Genoud, T. Graf, A. Junod, G. Triscone and J. Muller. Physica C 177 (1991) 315.

[56] T. Tsukamoto, G. Triscone, J.-Y. Genoud, K.-Q. Wang, E. Janod, A. Junod and J. Muller, J. Alloys and Comp., to be published.

[57] C. Opagiste, M. Couach, A.F. Khoder, T. Graf, A. Junod, G. Triscone, J. Muller, T.K. Jondo, J.-L. Jorda, R. Abraham, M.Th. CohenAdad, L.A. Bursill, O. Leckel and M.G. Blanchin, Physica C 205 (1993) 247.

[58] C. Opagiste, G. Triscone, M. Couach, T.K. Jondo, J.-L. Jorda, A. Junod, A.F. Khoder and J. Muller, Physica C 213 (1993) 17.

[59] C. Opagiste, G. Triscone, M. Couach, T. Jondo, J.-L. Jorda, A. Junod and A. Khoder, in: Proc. of LT-20 (1993), Eugene USA. 